\title{
EVERY SHIFT AUTOMORPHISM VARIETY HAS AN INFINITE SUBDIRECTLY IRREDUCIBLE MEMBER
}

\author{
KATE S. OWENS \\ (Received 17 February 2009; accepted 8 July 2009) \\ Communicated by M. G. Jackson
}

\begin{abstract}
A shift automorphism algebra is one satisfying the conditions of the shift automorphism theorem, and a shift automorphism variety is a variety generated by a shift automorphism algebra. In this paper, we show that every shift automorphism variety contains a countably infinite subdirectly irreducible algebra.
\end{abstract}

2000 Mathematics subject classification: primary 03C05; secondary 08B05, 08B26.

Keywords and phrases: shift automorphism algebra, shift automorphism variety, infinite subdirectly irreducible algebras.

We conceive of an algebra $\mathbf{A}$ as an ordered system $\left\langle A, F_{i}\right\rangle_{i \in I}$ where $A$ is a nonempty set and each $F_{i}$ is an operation on $A$ of finite rank for all $i \in I$. From $\mathbf{A}$, we can build $\operatorname{HSP}(\mathbf{A})$, the class containing all homomorphic images of subalgebras of direct powers of $\mathbf{A}$. If $\Sigma$ is the set of all equations true in $\mathbf{A}$, let $\mathcal{V}$ be the class of all algebraic structures satisfying all the equations in $\Sigma$. According to a famous result of Garrett Birkhoff, $\operatorname{HSP}(\mathbf{A})=\mathcal{V}$. In this case, we call $\mathcal{V}$ the variety generated by $\mathbf{A}$. If there is a finite list of equations true in an algebra from which all equations true in the algebra can be deduced, we say the algebra is finitely based. If no such finite list exists, we say the algebra is nonfinitely based.

A subdirect representation of $\mathbf{A}$ is a system $\left\langle h_{i}: i \in I\right\rangle$ of homomorphisms with domain $A$ so that the system of homomorphisms separates points: that is, provided for all $a, b \in A$ with $a \neq b$, there is an $i \in I$ so that

$$
h_{i}(a) \neq h_{i}(b) .
$$

The images $h_{i}(\mathbf{A})$ are called the subdirect factors of the subdirect representation. A subdirect representation is called trivial if one of the homomorphisms is one-toone. An algebra $\mathbf{A}$ is subdirectly irreducible provided every subdirect representation of $\mathbf{A}$ is trivial. The subdirectly irreducible algebras are exactly those which have a critical pair: a pair $(a, b)$ of distinct elements such that any homomorphism that is not

(C) 2010 Australian Mathematical Publishing Association Inc. 1446-7887/2010 \$16.00 
one-to-one must assign $a$ and $b$ the same value. According to another famous result of Birkhoff, every algebra $\mathbf{A}$ has a subdirect representation in which the subdirect factor algebras of the representation are subdirectly irreducible. As a consequence, every variety is determined by its subdirectly irreducible members.

According to a result of Robert Quackenbush, if a variety generated by a finite algebra has an infinite subdirectly irreducible member, it must also have arbitrarily large finite ones [17]. In 1981, Dziobiak improved this result by showing that the same holds in any locally finite variety [6]. A problem of Quackenbush asks whether there exists a finite algebra such that the variety it generates contains infinitely many subdirectly irreducible members but no infinite ones. One of the things McKenzie did in [11] was to provide an example of a four-element algebra of countable signature that generates a variety with this property. Whether or not this is possible with an algebra with only finitely many basic operations is not yet known.

Starting with a finite algebra $\mathbf{A}$, there is no guarantee that the variety generated by $\mathbf{A}$ is finitely based. Likewise, the variety generated by $\mathbf{A}$ is not guaranteed to have a finite upper bound on the size of its subdirectly irreducible members. In 1976, both Robert Park and Bjarni Jónnson proposed the following problem relating these three finiteness conditions.

The Park-Jónsson problem. Let $\mathbf{A}$ be a finite algebra with finitely many basic operations. If there exists a finite bound on the size of all the subdirectly irreducible members of the variety generated by $\mathbf{A}$, must $\mathbf{A}$ be finitely based?

Jónsson offered the above as an open problem at a seminar at Oberwolfach. Around the same time, Park proved in his $\mathrm{PhD}$ dissertation that the contrapositive held for all the then known examples of nonfinitely based finite algebras [15]. This paper extends Park's result to a particular infinite class of finite algebras known to be nonfinitely based.

An algebra is locally finite provided each of its finitely generated subalgebras is finite, and a variety $\mathcal{V}$ is locally finite if each of its algebras is locally finite. An element $0 \in A$ is called an absorbing element provided any operation evaluated at any tuple containing 0 outputs the value 0 . We say that an element of $A$ is proper if it is not the absorbing element, and we call an $n$-tuple $\left(a_{0}, \ldots, a_{n-1}\right)$ containing elements of $A$ proper if each $a_{i}$ is a proper element of $A$.

As Wald did in her doctoral dissertation [19], we define a shift automorphism algebra as an infinite, locally finite algebra with only finitely many fundamental operations, with an absorbing element 0 , and with an automorphism $\sigma$ such that:

(1) the only finite $\sigma$-orbit of $\mathbf{A}$ is $\{0\}$;

(2) the proper part of $F$ is partitioned by $\sigma$ into only finitely many orbits, for each fundamental operation $F$ of $\mathbf{A}$;

(3) there is a proper element $a$ of $A$ and a nonconstant unary polynomial function $f$ of A such that $f(a)=\sigma(a)$.

A shift automorphism variety is any variety generated by a shift automorphism algebra. This definition is slightly different to Wald's; she only required that a shift 
automorphism variety be a variety that contains a shift automorphism algebra. Notice that we have not stipulated that a shift automorphism algebra be uniformly locally finite. As shown in [4], this follows from the properties that are stipulated. The shift automorphism theorem (Theorem 1 in [4]) states that any shift automorphism algebra is inherently nonfinitely based and, under our definition, any shift automorphism variety is inherently nonfinitely based.

In 1951, Lyndon demonstrated that all two-element algebras are finitely based [8]. Lyndon's result relied on an analysis of work done by Post [16] in 1941. In 1980, Berman [5] proved Lyndon's result using Baker's finite basis theorem [2] and a finite basis theorem for certain congruence permutable varieties, proven in 1978, by McKenzie [10]. In the other direction, many kinds of finite algebras have been shown to be nonfinitely based. The earliest example, constructed by Lyndon in 1954, is a seven-element groupoid [9]. Although it is nonfinitely based, it fails to be inherently nonfinitely based; see [1]. In 1965, Murskil created a three-element nonfinitely based groupoid [13] which he later showed to be inherently nonfinitely based [14]. For some interesting examples of finite algebras proven to be nonfinitely based with help of the shift automorphism theorem, see [3, 7, 18-20].

Our aim here is to show that if $\mathbf{A}$ is a shift automorphism algebra, then $\mathbf{A}$ generates a variety with a subdirectly irreducible algebra that is countably infinite. Hence we provide an affirmative answer to the Park-Jónsson problem for an infinite subclass of all algebras known to be nonfinitely based. According to the analysis done by Murskii in [14], those finite groupoids which generate varieties containing shift automorphism algebras account for a nontrivial fraction of all nonfinitely based groupoids. Thus if there are finite algebras that provide negative answers to the Park-Jónsson problem, they would be less common. Indeed, Willard has offered a 50 euro reward for the first such example [21].

THEOREM. Let $\mathcal{V}$ be a shift automorphism variety. Then $\mathcal{V}$ contains a countably infinite subdirectly irreducible algebra.

PROOF. Let $\mathbf{A}$ be a shift automorphism algebra that generates $\mathcal{V}$. In order to construct a subdirectly irreducible algebra in $\mathcal{V}$, we begin by making a congruence $\theta$ of $\mathbf{A}$. The congruence $\theta$ is chosen to be a maximal congruence with respect to separating $a$ and 0 , where $a$ is the element identified in condition (3) above. The quotient algebra $\mathbf{A} / \theta$ will be subdirectly irreducible, and provided it is of sufficient size, our construction will be complete. Thus we aim to show that $\theta$ separates countably many pairs of points. As pointed out in [12], every shift automorphism algebra has a countably infinite subalgebra that is also a shift automorphism algebra. Thus it is harmless to assume that $\mathbf{A}$ is countably infinite.

The polynomial function $f(x)$ has a property worth mentioning. It has an associated term function that can be described using a term tree. Let $t^{\mathbf{A}}\left(x, y_{1}, \ldots, y_{k-1}\right)$ be a term function of $\mathbf{A}$ so that

$$
f(x)=t^{\mathbf{A}}\left(x, c_{1}, \ldots, c_{k-1}\right)
$$


where each $c_{i} \in A$. Let $t^{\mathbf{A}}(x, \bar{c})$ denote this term function. Note that in a shift automorphism algebra $\mathbf{A}$ every constant symbol $d$ must denote the absorbing element 0 . In particular, none of these constant symbols can occur in $t^{\mathbf{A}}(x, \bar{y})$.

Claim 1. The term $t(x, \bar{y})$ as mentioned above contains an operation symbol of rank at least 2.

PROOF. With the aim of reaching a contradiction, we begin by supposing $t(x, \bar{y})$ contains only unary operation symbols. Recall that the $\sigma$-orbit of $a$ is infinite and also that the algebra $\mathbf{A}$ is locally finite. Notice that $f(a)=\sigma(a)$ implies that

$$
\begin{aligned}
f(f(a)) & =f(\sigma(a)) \\
& =\sigma(f(a)) \\
& =\sigma(\sigma(a)) \\
& =\sigma^{2}(a) .
\end{aligned}
$$

Likewise,

$$
\begin{aligned}
f(f(f(a))) & =f\left(\sigma^{2}(a)\right) \\
& =\sigma^{2}(f(a)) \\
& =\sigma^{2}(\sigma(a)) \\
& =\sigma^{3}(a) .
\end{aligned}
$$

Indeed, given that $f^{m}(a)=\sigma^{m}(a)$,

$$
\begin{aligned}
f^{m+1}(a) & =f\left(f^{m}(a)\right) \\
& =f\left(\sigma^{m}(a)\right) \\
& =\sigma^{m}(f(a)) \\
& =\sigma^{m}(\sigma(a)) \\
& =\sigma^{m+1}(a) .
\end{aligned}
$$

Thus the countably infinite set $\left\{\sigma^{m}(a) \mid m \in \omega\right\}$ can be generated by the element $a$ and applying one-place operations repeatedly. This contradicts the assumption that the algebra $\mathbf{A}$ is locally finite.

In order to proceed, we need to make use of some machinery found in the proof of the shift automorphism theorem. Additional details of this machinery can be found in [4] and [12]. We say that two elements are operationally related provided they are entries in a tuple of proper elements that belongs to some fundamental operation of $\mathbf{A}$. Given a subalgebra $\mathbf{S}$ of $\mathbf{A}$, we say that $\mathbf{S}$ is $\sigma$-decomposable if there is a subalgebra $\mathbf{S}_{0}$ (called the core of the decomposition) such that

$$
S=\bigcup_{i \in \mathbb{Z}} \sigma^{i}\left(S_{0}\right)
$$


and no element of $\mathbf{S}_{0}$ is operationally related to any others in any nontrivial $\sigma$ translate of $S_{0}$. Note that the $\sigma$-decomposition is constructed so that elements from different blocks cannot be operationally related; that is, if $F$ is any basic operation and $s_{0}, \ldots, s_{\ell-1} \in S$ such that

$$
F\left(s_{0}, \ldots, s_{\ell-1}\right) \neq 0,
$$

we are guaranteed that $\left\{s_{0}, \ldots, s_{\ell-1}\right\} \subset \sigma^{j}\left(S_{0}\right)$ for some unique integer $j$.

Some of the subalgebras of $\mathbf{A}$ might turn out to be $\sigma$-decomposable while others might not. We need more control of the decomposable subalgebras. Let $n$ be a natural number. The methods in [4] and [12, Claim 2] show how to replace the automorphism $\sigma$ by an automorphism $\tau$ and the polynomial function $f(x)$ by another polynomial function $g(x)$ so that $\tau$ and $g(x)$ still show that $\mathbf{A}$ is a shift automorphism algebra and each subalgebra of $\mathbf{A}$ generated by the union of any $n$-many $\tau$-orbits is $\tau$-decomposable.

Indeed, $\tau$ turns out to be a power (depending on $n$ ) of the automorphism $\sigma$. For our purposes, we pick $n$ to be larger than the ranks of the basic operations of $\mathbf{A}$. In order to conserve notation, we suppose that each subalgebra of $\mathbf{A}$ generated by the union of any $n$-many $\sigma$-orbits is already $\sigma$-decomposable.

We will choose the generating orbits for the $\sigma$-decomposable subalgebra in a particular way, as discussed below. The individual pieces of the $\sigma$-decomposition will be called blocks.

We saw above that the polynomial function $f(x)$ has the associated term function $t^{\mathbf{A}}(x, \bar{y})$ with a tuple $\bar{c}$ consisting of elements from $\mathbf{A}$ and where $t^{\mathbf{A}}(x, \bar{c})=f(x)$. Furthermore, we know that $t^{\mathbf{A}}(a, \bar{c})=\sigma(a)$. This implies that

$$
\begin{aligned}
a & =\sigma^{-1}\left(t^{\mathbf{A}}(a, \bar{c})\right) \\
& =t^{\mathbf{A}}\left(\sigma^{-1}(a), \sigma^{-1}(\bar{c})\right)
\end{aligned}
$$

where $\sigma^{-1}(\bar{c})$ denotes $\sigma^{-1}\left(c_{1}\right), \ldots, \sigma^{-1}\left(c_{k-1}\right)$. At some leaf in the evaluation tree whose output is $a$, we find the element $\sigma^{-1}(a)$.

From our argument in Claim 1, we know that by starting at $\sigma^{-1}(a)$ and following the evaluation tree upward, after only finitely many applications of unary operations, we will come to an operation $Q^{\mathbf{A}}$ of rank at least 2, as seen in Figure 1 (below). Let $H$ denote this string of unary operation symbols and let $H^{\mathbf{A}}$ be the function obtained by composing the unary operations denoted by the unary operation symbols occurring in the string $H$.

Thus on the evaluation tree, under the (at least binary) operation $Q^{\mathbf{A}}$ we find at least one node labeled $H^{\mathbf{A}}\left(\sigma^{-1}(a)\right)$. Furthermore, since $Q$ is an operation symbol from $\mathbf{A}$, it has rank smaller than $n$. Since $Q^{\mathbf{A}}$ is not unary, it has at least one other input distinct from $H^{\mathbf{A}}\left(\sigma^{-1}(a)\right)$; call these other inputs $b_{1}, \ldots, b_{r-1}$ where $r \geq 2$ is the rank of $Q$.

In order to alleviate notation, we will denote $Q^{\mathbf{A}}\left(H^{\mathbf{A}}\left(\sigma^{-1}(a)\right), b_{1}, \ldots, b_{r-1}\right)$ by $Q^{\mathbf{A}}\left(H^{\mathbf{A}}\left(\sigma^{-1}(a)\right), \bar{b}\right)$. The $\sigma$-decomposable subalgebra of $\mathbf{A}$ that is of interest to us is the subalgebra generated by the orbits of these leaf elements; namely the orbits of the elements $\sigma^{-1}(a), b_{1}, \ldots, b_{r-1}$. 


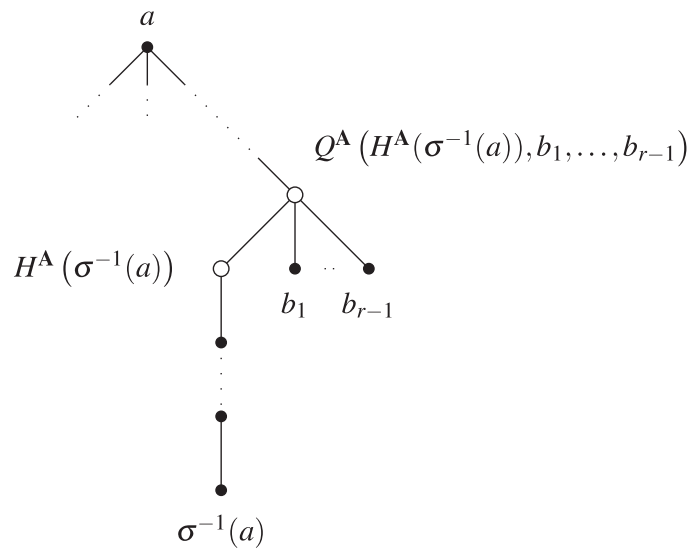

FIGURE 1. The evaluation tree of the polynomial $f(x)$.

Claim 2. Given any natural number $k$, if $\sigma^{-k}(a) \theta 0$, then $a \theta 0$.

PROOF. We have seen that $a$ can be written as the output of a term function which contains the input $\sigma^{-1}(a)$. In this case, we will say that $\sigma^{-1}(a)$ is a factor of $a$ or just that $\sigma^{-1}(a)$ divides $a$. In general, an element $r$ divides an element $q$ if there is an evaluation tree whose output is $q$ and which contains a leaf with the label $r$. Now,

$$
\begin{gathered}
a=t^{\mathbf{A}}\left(\sigma^{-1}(a), \sigma^{-1}(\bar{c})\right) \\
\Downarrow \\
\sigma^{-1}(a)=t^{\mathbf{A}}\left(\sigma^{-2}(a), \sigma^{-2}(\bar{c})\right)
\end{gathered}
$$

and so $\sigma^{-2}(a)$ divides $\sigma^{-1}(a)$. Combining these, we see that

$$
a=t^{\mathbf{A}}\left(t^{\mathbf{A}}\left(\sigma^{-2}(a), \sigma^{-2}(\bar{c})\right), \sigma^{-1}(\bar{c})\right)
$$

and so $\sigma^{-2}(a)$ divides $a$. In general, since

$$
\sigma^{-k}(a)=t^{\mathbf{A}}\left(\sigma^{-(k+1)}(a), \sigma^{-(k+1)}(\bar{c})\right)
$$

by back-substituting we see that $\sigma^{-k}(a)$ also divides $a$.

Now suppose that $\sigma^{-k}(a) \theta 0$. Since $\sigma^{-k}(a)$ divides $a$, it is a node on an evaluation tree whose output is $a$. The top node of any evaluation tree with a node that is $\theta$-related to 0 is itself $\theta$-related to 0 . This occurs since 0 is an absorbing element and it is found inside a tuple of an operation symbol of $\mathbf{A}$. Thus if $\sigma^{-k}(a)$ is $\theta$-related to 0 , it follows that $a \theta 0$.

Our aim is the following: given any natural number $m$, the element $\sigma^{-m}(a)$ is separated by $\theta$ from $\sigma^{-q}(a)$ for any natural number $q \neq m$. The proof of this proceeds by contradiction. 
Claim 3. Let $m$ and $q$ be distinct natural numbers. Then

$$
\sigma^{-m}(a) \theta \sigma^{-q}(a) \Longrightarrow \sigma^{-(m-1)}(a) \theta 0 .
$$

PROOF. We begin by supposing that $\sigma^{-m}(a)$ and $\sigma^{-q}(a)$ are related by $\theta$. Since $\sigma$ is an automorphism, we can apply $\sigma^{-1}$ to the evaluation tree above by applying it to each leaf. This produces an evaluation tree whose output is $\sigma^{-1}(a)$ and which has the node

$$
Q^{\mathbf{A}}\left(H^{\mathbf{A}}\left(\sigma^{-2}(a)\right), \sigma^{-1}(\bar{b})\right) .
$$

Repeating this process, the element $\sigma^{-(m-1)}(a)$ is the output of an evaluation tree which has the node

$$
Q^{\mathbf{A}}\left(H^{\mathbf{A}}\left(\sigma^{-m}(a)\right), \sigma^{-(m-1)}(\bar{b})\right) .
$$

Since $\sigma^{-q}(a)$ and $\sigma^{-m}(a)$ are $\theta$-related, we know that

$$
H^{\mathbf{A}}\left(\sigma^{-m}(a)\right) \theta H^{\mathbf{A}}\left(\sigma^{-q}(a)\right)
$$

since $H$ is a composition of unary operation symbols. Consider the following.

$$
\begin{array}{ccl}
H^{\mathbf{A}}\left(\sigma^{-m}(a)\right) & \theta & H^{\mathbf{A}}\left(\sigma^{-q}(a)\right) \\
\sigma^{-(m-1)}\left(b_{1}\right) & \theta & \sigma^{-(m-1)}\left(b_{1}\right) \\
\vdots & & \vdots \\
\sigma^{-(m-1)}\left(b_{r-1}\right) & \theta & \sigma^{-(m-1)}\left(b_{r-1}\right) \\
\hline Q^{\mathbf{A}}\left(H^{\mathbf{A}}\left(\sigma^{-m}(a)\right), \sigma^{-(m-1)}(\bar{b})\right) & \theta & Q^{\mathbf{A}}\left(H^{\mathbf{A}}\left(\sigma^{-q}(a)\right), \sigma^{-(m-1)}(\bar{b})\right) .
\end{array}
$$

The right-hand-side term $Q^{\mathbf{A}}\left(H^{\mathbf{A}}\left(\sigma^{-q}(a)\right), \sigma^{-(m-1)}(\bar{b})\right)$ contains inputs from two different $\sigma$-decomposition blocks, so it must evaluate to the default element 0 . Meanwhile, the left-hand side is a factor of $\sigma^{-(m-1)}(a)$. Since $\theta$ is a congruence, this implies that $\sigma^{-(m-1)}(a)$ is $\theta$-related to 0 .

As $a$ and 0 are not related by $\theta$, we have shown that

$$
\left(\sigma^{-m}(a), \sigma^{-q}(a)\right) \notin \theta
$$

for any distinct natural numbers $m$ and $q$. The quotient algebra $\mathbf{A} / \theta$ will have countably infinitely many elements and it is subdirectly irreducible by the maximality of $\theta$ with respect to separating $a$ and 0 . Furthermore, it is in $\mathcal{V}$.

\section{References}

[1] T. Bajusz, G. F. McNulty and Á. Szendrei, 'Lyndon's groupoid is not inherently nonfinitely based', Algebra Universalis 27(2) (1990), 254-260.

[2] K. A. Baker, 'Finite equational bases for finite algebras in a congruence-distributive equational class', Adv. Math. 24(3) (1977), 207-243.

[3] K. A. Baker, G. F. McNulty and H. Werner, 'The finitely based varieties of graph algebras', Acta Sci. Math. (Szeged) 51(1-2) (1987), 3-15. 
[4] K. A. Baker, G. F. McNulty and H. Werner, 'Shift-automorphism methods for inherently nonfinitely based varieties of algebras', Czechoslovak Math. J. 39(114)(1) (1989), 53-69.

[5] J. Berman, 'A proof of Lyndon's finite basis theorem', Discrete Math. 29(3) (1980), 229-233.

[6] W. Dziobiak, 'On infinite subdirectly irreducible algebras in locally finite equational classes', Algebra Universalis 13(3) (1981), 393-394.

[7] K. Kearnes and R. Willard, 'Inherently nonfinitely based solvable algebras', Canad. Math. Bull. 37(4) (1994), 514-521.

[8] R. C. Lyndon, 'Identities in two-valued calculi', Trans. Amer. Math. Soc. 71 (1951), 457-465.

[9] R. C. Lyndon, 'Identities in finite algebras', Proc. Amer. Math. Soc. 5 (1954), 8-9.

[10] R. McKenzie, 'Para primal varieties: a study of finite axiomatizability and definable principal congruences in locally finite varieties', Algebra Universalis 8(3) (1978), 336-348.

[11] R. McKenzie, 'The residual bounds of finite algebras', Internat. J. Algebra Comput. 6 (1996), $1-28$.

[12] G. F. McNulty, Z. Székely and R. Willard, 'Equational complexity of the finite algebra membership problem', Internat. J. Algebra Comput. 18 (2008), 1283-1319.

[13] V. L. Murskiı̌, 'The existence in three-valued logic of closed class with finite basis not having a finite complete set of identities', English Translation Soviet Math. Dokl. 6 (1965), 1020-1024.

[14] V. L. Murskiü, 'The number of $k$-element algebras with a binary operation which do not have a finite basis of identities', Problemy Kibernet. (1979), 5-27.

[15] R. E. Park, 'Equational classes on non-associative ordered algebras', PhD Thesis, University of California, Los Angeles, 1976.

[16] E. L. Post, The Two-Valued Iterative Systems of Mathematical Logic, Annals of Mathematics Studies, 5 (Princeton University Press, Princeton, NJ, 1941).

[17] R. W. Quackenbush, 'Equational classes generated by finite algebras', Algebra Universalis 1 (1971/72), 265-266.

[18] Á. Szendrei, 'Non-finitely based finite groupoids generating minimal varieties', Acta Sci. Math. (Szeged) 57(1-4) (1993), 593-600.

[19] L. A. Wald, 'Minimal inherently nonfinitely based varieties of groupoids' PhD Thesis, University of California, Los Angeles, 1998.

[20] B. L. Walter, 'The finitely based varieties of looped directed graph algebras', Acta Sci. Math. (Szeged) 72(3-4) (2006), 421-458.

[21] R. Willard, 'The finite basis problem', in: Contributions to General Algebra, Vol. 15 (Heyn, Klagenfurt, 2004), pp. 199-206.

\section{KATE S. OWENS, Department of Mathematics, University of South Carolina, 1523 Greene Street, Columbia, SC 29208, USA \\ e-mail: scottkh@mailbox.sc.edu}

